\title{
The role of APC/C inhibitor Emi2/XErp1 in oscillatory dynamics of early embryonic cell cycles
}

\author{
P.K. Vinod ${ }^{a}$, Xin Zhou ${ }^{\text {a }}$, Tongli Zhang ${ }^{a}$, Thomas U. Mayer ${ }^{b}$, Bela Novak ${ }^{\mathrm{a}, \mathrm{c}, *}$ \\ a Oxford Centre for Integrative Systems Biology, Department of Biochemistry, University of Oxford, South Parks Road, Oxford OX1 3QU, UK \\ ${ }^{b}$ Department of Biology and Konstanz Research School Chemical Biology, University of Konstanz, Universitätsstr. 10, 78457 Konstanz, Germany \\ ' Budapest University of Technology and Economics, 1111 Budapest, Szt. Gellert ter 4, Hungary
}

\section{H I G H L I G H T S}

- Modelling the interplay of $\mathrm{Cdk} 1: \mathrm{CycB}$, Cdc20, APC/C, Emi2 and PP2A-B'56 in the control of early embryonic cell cycle - The period and amplitude of early embryonic cell cycle are sensitive to Emi2 levels.

- Emi2 interferes with intrinsic time delay in APC/C activation and inactivation.

\section{Keywords:}

Embryonic cell cycle

Oscillation

Mathematical modelling

Cyclin-B

Anaphase Promoting Complex

Cyclosome
GRAPHICAL ABSTRACT

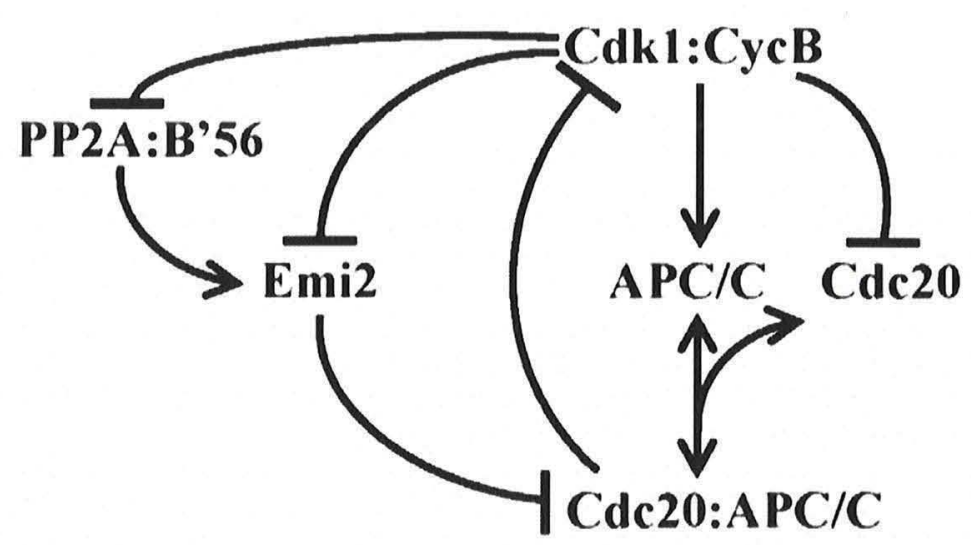

\begin{abstract}
A B S T R A C T
The early embryonic Xenopus cell cycles are characterized by alternating oscillations of Cyclin-dependent kinase-1 (Cdk1) and Anaphase Promoting Complex/Cyclosome (APC/C) activities. The early cycles before midblastula transition lack significant inhibitory Cdk1 phosphorylations and are driven by periodic accumulation of Cyclin B before M phase and its degradation by APC/C at the end of $M$ phase. Both experiments and mathematical modelling suggest that while $C \mathrm{dk} 1: \mathrm{CycB}$ phosphorylation activates $A P C / C$, it inhibits its co-activator Cdc20 (Fizzy). These interactions create an amplified negative-feedback loop which is at the heart of all cell cycle oscillations. Recent experiments find that the APC/C inhibitor, Emi2/XErp1 is essential for large amplitude and short period Cyclin B oscillations during early divisions in the intact Xenopus embryo. This finding is counter-intuitive since larger amplitudes should come with slower cycle times. We explain this paradox by analysing the amplified negative feedback model extended with APC/C inhibition by Emi2. We show that Emi2 interferes with the intrinsic time-delay in APC/C activation and inactivation to increase the amplitude as well as shorten the period of Cyclin B oscillation.
\end{abstract}

* Corresponding author at: Oxford Centre for Integrative Systems Biology, Department of Biochemistry, University of Oxford, South Parks Road, Oxford OX1 3QU, UK

E-mail address: bela.novak@bioch.ox.ac.uk (B. Novak). 


\section{Introduction}

Metaphase II arrested Xenopus eggs upon fertilization complete meio sis and undergo early embryonic cell cycles marked by periodic activation and inactivation of cyclin dependent kinase (Cdk1). The length of first division is prolonged ( $90 \mathrm{~min}$ ) in comparison to subsequent eleven cycles which are rapid $(\sim 30 \mathrm{~min})$ synchronous cell divisions [1,2]. During these rapid cell cycles, embryos alternate between $\mathrm{S}$ phase (DNA replication) and $\mathrm{M}$ phase (karyokinesis/mitosis and cytokinesis) without cell growth and any intervening gap (G1 and G2) phases. At the end of twelfth cycle the embryos undergo midblastula transition (MBT) when the cell cycle lengthens with addition of gap phases and the activation of transcription and checkpoint mechanism [3].

During the first cycle the activity of Cdk1 is regulated by the accumulation and destruction of its activator Cyclin B and also by inhibitory phosphorylations and dephosphorylations. However, during cycles 2-12 inhibitory phosphorylations of Cdk1 are not detectable in the intact embryo [3-5]. The destruction of Cyclin B $(\mathrm{CycB})$ at the end of mitosis is regulated by Anaphase Promoting Complex/Cyclosome (APC/C), an ubiquitin ligase that targets its substrates to destruction by proteasome. The temporal control of APC/C activity depends on its phosphorylation state as well as on the regulation of its co-activators and inhibitors [6,7]. Cdk1:CycB is known to activate $\mathrm{APC} / \mathrm{C}$ by phosphorylating its several subunits and $\mathrm{APC} / \mathrm{C}$ in turn terminates the Cdk1 activity by mediating Cyclin B destruction. As a consequence, $\mathrm{Cdk} 1: \mathrm{CycB}$ and $\mathrm{APC} / \mathrm{C}$ form the core negative feedback loop. $\mathrm{APC} / \mathrm{C}$ by itself is unable to ubiquitinate Cyclin B and it requires its co-activators such as $\mathrm{Cdc} 20$ and $\mathrm{Cdh} 1$ that help in recognizing its substrates. In Xenopus embryos, Cdc20 role as an APC/C activator is well established, while Cdh1 is barely detectable [8,9]. Cdc20 is also regulated by $\mathrm{Cdk} 1: \mathrm{CycB}$ dependent phosphorylation in such a way that only the dephosphorylated form can bind and activate phosphorylated APC/C [10-12].

In the early embryonic cell cycle of Xenopus, APC/C is not subjected to inhibition by mitotic checkpoint proteins and by early mitotic inhibitor 1 , Emi1 $[13,14]$. The other APC/C inhibitor Emi2 (XErp1) is known to be responsible for the metaphase II arrest in meiosis $[15,16]$. Emi2 is degraded at fertilization allowing APC/C activation and exit from metaphase II arrest $[17,18]$. Although it is known that Emi2 re-accumulates after fertilization and exists until MBT, its functional role during early embryonic cell cycle was unknown [19]. Recently, Mayer and his colleagues reported that Emi2 (XErp1) is required for large amplitude and short period Cyclin B oscillations during early mitotic divisions in the intact embryo [20]. Even more, Emi2 depletion leads to embryonic lethality [20].

In this work, we extend a model of the early embryonic Xenopus cell cycle with regulation of Emi2 and analyse its dynamical effect on Cyclin B oscillations. With this extended mathematical model we provide an explanation for seemingly counter-intuitive observations that an APC/C inhibitor speeds up the oscillation and increases its amplitude.

\section{Results and discussion}

\subsection{A mathematical model of early division cycles in Xenopus embryos}

Ciliberto et al. [12] proposed a mathematical model of cell cycle control in Xenopus egg extracts based on inhibitory tyrosine phosphorylation of Cdk1 and Cyclin B destruction by Cdc20:APC (APC/C bound to its co-activator $\mathrm{Cdc} 20$ ). Since intact embryos lack significant inhibitory phosphorylation of Cdk1 during cycles 2-12, these oscillations must be driven primarily by Cdc20:APC dependent periodic Cyclin $B$ degradation. Therefore in our subsequent analysis we excluded the inhibitory Cdk1 phosphorylation without loss of generality. Negative feedback loop with sufficient time-delay is a necessary requirement for any oscillations [21]. These two requirements are achieved in the Ciliberto's model by dual control of Cdk1:CycB on Cdc20:APC. Cdk1:CycB is activating APC/C and inactivating Cdc20 through phosphorylation (Fig. 1). The active Cdc20:APC-P complex can be formed only when APC/C is phosphorylated (APC-P) by Cdk1:CycB and Cdc20 is dephosphorylated. The Cdc20:APC-P control network is characterized by a negative and a double-negative feedback loop. The negative feedback loop arises because Cdk1:CycB activates the APC/C component of Cdc20:APC-P which promotes the degradation of Cyclin $B$ $(\mathrm{Cdk} 1: \mathrm{CycB} \rightarrow \mathrm{APC}-\mathrm{P} \rightarrow \mathrm{Cdc} 20: \mathrm{APC}-\mathrm{P} \dashv \mathrm{Cdk} 1: \mathrm{CycB})$. The doublenegative feedback loop is the consequence of $\mathrm{Cdc} 20$ inhibitory phosphorylation by Cdk1: $\mathrm{CycB}(\mathrm{Cdk} 1: \mathrm{CycB} \dashv \mathrm{Cdc} 20 \rightarrow \mathrm{Cdc} 20: \mathrm{APC}-$ $\mathrm{P} \dashv \mathrm{Cdk1}: \mathrm{CycB}$ ). This double-negative feedback loop operates as a bistable switch and it provides the required time-delay for the negative feedback loop to oscillate. A further requirement for this type of control network to oscillate is that the double-negative feedback must operate on a faster time-scale than negative feedback. This implies that $\mathrm{Cdc} 20$ has to be phosphorylated and dephosphorylated faster than APC/C. The oscillatory behaviour of the Ciliberto's model (see Table 1) without inhibitory Cdk1 phosphorylation is illustrated on Fig. 2A. The kinetic parameter values were chosen (see Table 2 \& Methods) such that the period of oscillation ( $37 \mathrm{~min}$ ) matches the length of cell division reported by Tischer et al. [20] for Emi2 depleted embryos.

At the beginning of a cycle when the concentration of Cyclin B is low, the inhibitory phosphorylation of $\mathrm{Cdc} 20$ by $\mathrm{Cdk} 1: \mathrm{CycB}$ precedes the activatory phosphorylation of APC/C (Fig. 2A). This limits the formation of active Cdc20:APC-P complex leading to rise in Cdk1: $\mathrm{CycB}$ activity with the accumulation of Cyclin B. Later the rise of phosphorylated APC/C (APC-P) with increasing Cdk1:CycB activity overrides the negative effect on $\mathrm{Cdc} 20$ to generate active Cdc20:APC-P complexes, which initiate Cyclin B destruction. With decreasing Cyclin $B$ level the Cdk1 activity drops and Cdc20 is dephosphorylated faster than APC/C. Therefore the concentration of active Cdc20:APC-P complex increases until Cdk1: $\mathrm{CycB}$ drops to a low level when phosphorylation of $\mathrm{APC} / \mathrm{C}$ becomes compromised.

\subsection{The effect of Emi2 on Cyclin B oscillations}

To study the role of Emi2 in early embryonic cell cycles, Ciliberto's model was supplemented with Emi2 regulation (see Table 1). Emi2 levels remain constant $(E m i 2 \mathrm{~T})$ during early cell division cycles, however its activity is inhibited by $\mathrm{Cdk} 1: \mathrm{CycB}$ dependent phosphorylation and activated by PP2A-B'56 dependent dephosphorylation [20] Inactivation of $\mathrm{APC} / \mathrm{C}$ inhibitor $\mathrm{Emi} 2$ by $\mathrm{Cdk} 1: \mathrm{CycB}$ creates a three component negative feedback loop: $\mathrm{Cdk} 1: \mathrm{CycB} \dashv \mathrm{Emi} 2 \dashv \mathrm{Cdc} 20$ :APC $P \dashv \mathrm{Cdk1}$ :CycB. The dephosphorylated form of Emi2 binds to the active Cdc20:APC-P complex and inactivates it (Fig. 1). Notice that both $\mathrm{APC} / \mathrm{C}$ and its regulators (Cdc20 and Emi2) are controlled by Cdk1: СycB dependent phosphorylation and dephosphorylation catalysed by phosphatases. Tischer et al. [20] hypothesized that Emi2 regulation must ensure that it does not get prematurely activated when the Cdk1: $\mathrm{CycB}$ activity decreases during mitotic exit. In order to implement this idea in the model we assume that the phosphatase activating Emi2 is inactivated by $\mathrm{Cdk} 1: \mathrm{CycB}$ dependent phosphorylation. Although there is no direct evidence for $\mathrm{Cdk} 1: \mathrm{CycB}$ dependent regulation of $\mathrm{PP} 2 \mathrm{~A}-\mathrm{B}^{\prime}$ 56 , other forms of PP2A (and PP1 as well) are shown to be under the control of $\mathrm{Cdk} 1: \mathrm{CycB}$ activity [22-25]. Observe that the Emi2 phosphorylation state is regulated directly and indirectly (through PP2A-B'56) by Cdk1:CycB which creates a coherent feed-forward loop in the network.

Emi2 shortens the period of oscillations of Ciliberto's model from $37 \mathrm{~min}$ to $\sim 30 \mathrm{~min}$ (compare Fig. $2 \mathrm{~A} \mathrm{\&} \mathrm{B}$ ). During these oscillations active Emi2 (unphosphorylated) level starts to increase when the $\mathrm{Cdk1:CycB}$ concentration is very low and continues to increase until it gets inhibited by the fast rising $\mathrm{Cdk} 1: \mathrm{CycB}$ activity. In the model, the rate of Emi2 phosphorylation by $\mathrm{Cdk} 1: \mathrm{CycB}$ is slower than 


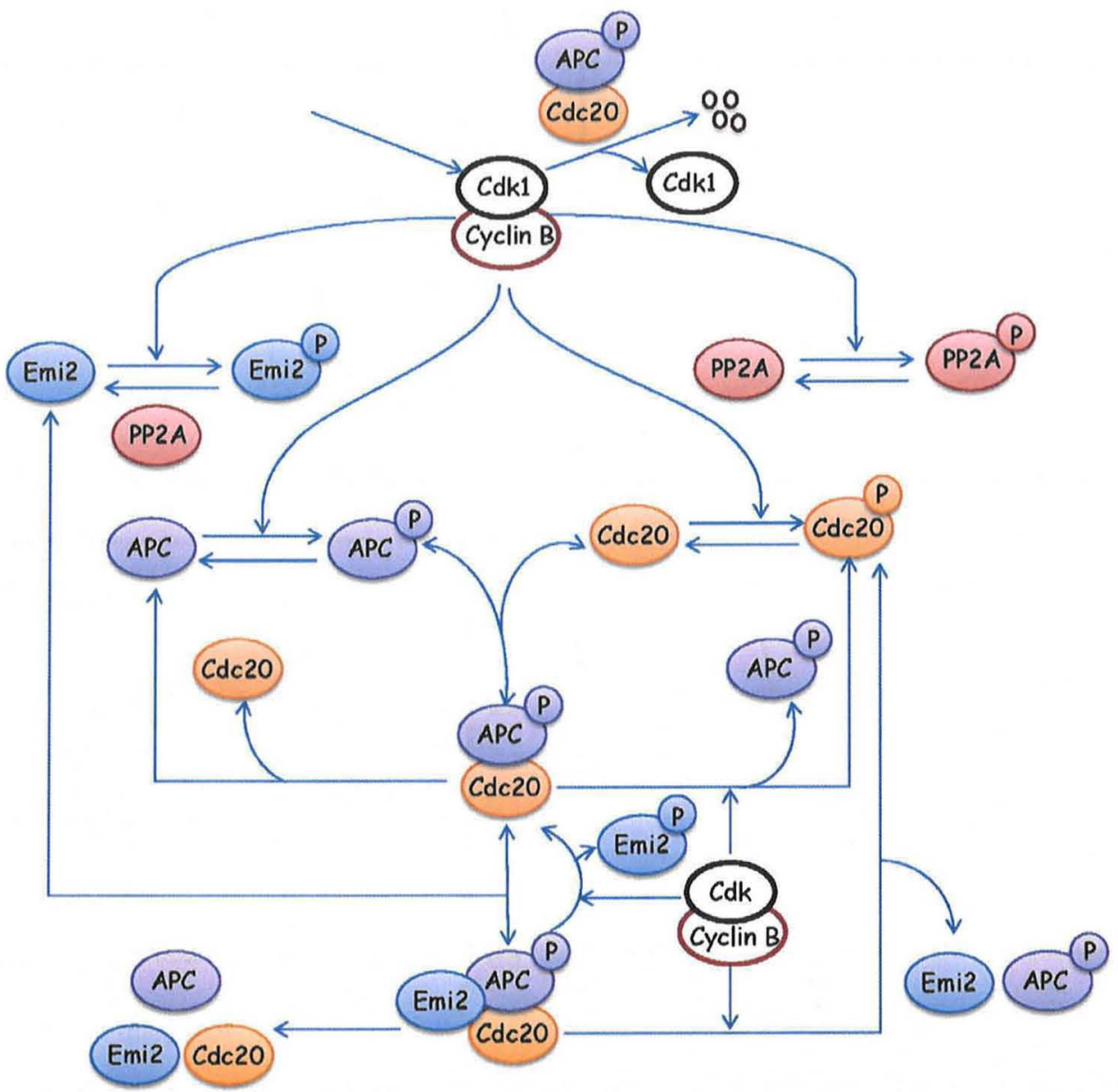

Fig. 1. The regulatory network involved in the control of early embryonic cell cycle of Xenopus. Cdk1 activity is controlled by Cyclin B synthesis and degradation. APC/C is activated by Cdk1:CycB phosphorylation, while Cdc20, Emi2 and PP2A are inactivated. The complex formation between phosphorylated APC/C (APC-P) and dephosphorylated Cdc20 (Cdc20: APC-P) is required to target Cyclin B for degradation by proteasome. The dephosphorylation of Emi2 by PP2A is required for its binding to Cdc20:APC-P complex to form inhibitory Cdc20:APC-P:Emi2 complex. The dissociation of all the complexes depends upon either inhibitory phosphorylations or dephosphorylations.

Table 1

Model equations.

(1) $\frac{\mathrm{d}[\mathrm{Cdk1}: \mathrm{CycB}]}{\mathrm{dt}}=k_{\mathrm{Cycs}}-k_{\mathrm{Cycd}} \cdot[\mathrm{Cdk} 1: \mathrm{CycB}]-k_{\mathrm{Cycd}} \cdot[\mathrm{Cdc} 20: \mathrm{APC}-\mathrm{P}] \cdot \frac{[\mathrm{Cdk} 1: \mathrm{CycB}]}{\sqrt{\mathrm{Cyc}}+[\mathrm{Cdk} 1: \mathrm{CyCB})}$

(2) $\frac{\mathrm{d}\left[\mathrm{APC}-\mathrm{P}_{\mathrm{T}}\right]}{\mathrm{dt}}=k_{\mathrm{Apca}} \cdot[\mathrm{Cdk} 1: \mathrm{CycB}] \cdot\left(\left[\mathrm{APC}_{\mathrm{T}}\right]-\left[\mathrm{APC}-\mathrm{P}_{\mathrm{T}}\right]\right)-k_{\mathrm{Apci}} \cdot\left[\mathrm{APC}-\mathrm{P}_{\mathrm{T}}\right]$

(3) $\frac{\mathrm{d}\left[\mathrm{Cdc} 20_{\mathrm{T}}^{\mathrm{np}}\right]}{\mathrm{d} t}=k_{\mathrm{Cdc} 20 \mathrm{a}} \cdot\left(\left[\mathrm{Cdc} 20_{\mathrm{T}}\right]-\left[\mathrm{Cdc} 20_{\mathrm{T}}^{\mathrm{np}}\right]\right)-k_{\mathrm{Cdc} 20 \mathrm{i}^{\circ}} \cdot[\mathrm{Cdk} 1: \mathrm{CycB}] \cdot\left[\mathrm{Cdc} 20_{\mathrm{T}}^{\mathrm{np}}\right]$

(4) $\frac{\mathrm{d}\left[\mathrm{Emi2} 2_{\mathrm{T}}^{\mathrm{A}}\right]}{\mathrm{dt}}=k_{\mathrm{Emi2a}} \cdot[\mathrm{PP} 2 \mathrm{~A}] \cdot\left(\left[\mathrm{Emi2} 2_{\mathrm{T}}\right]-\left[\mathrm{Emi2}_{\mathrm{T}}^{\mathrm{A}}\right]\right)-k_{\mathrm{Emi2i}} \cdot[\mathrm{Cdk} 1: \mathrm{CycB}] \cdot\left[\mathrm{Emi}_{\mathrm{T}}^{\mathrm{A}}\right]$

(5) $\frac{\mathrm{d}[\mathrm{PP} 2 \mathrm{~A}]}{d t}=k_{\mathrm{Pp} 2 \mathrm{aa}} \cdot\left(\left[\mathrm{PP} 2 \mathrm{~A}_{\mathrm{T}}\right]-[\mathrm{PP} 2 \mathrm{~A}]\right)-\left(k_{\mathrm{Pp} 2 \mathrm{ai}}+k_{\mathrm{Pp} 2 \mathrm{ai}} \cdot[\mathrm{Cdk} 1: \mathrm{CycB}]\right) \cdot[\mathrm{PP} 2 \mathrm{~A}]$

(6) $\frac{\mathrm{d}[\mathrm{Cdc} 20: \mathrm{APC}-\mathrm{P}]}{\mathrm{d} t}=k_{\mathrm{Apcass}} \cdot[\mathrm{APC}-\mathrm{P}] \cdot[\mathrm{Cdc} 20]+\left(k_{\text {Emi2diss }}+k_{\text {Emi2i }} \cdot[\mathrm{Cdk} 1: \mathrm{CycB}]\right)$ $\times[\mathrm{Cdc} 20: \mathrm{APC}-\mathrm{P}: \mathrm{Emi} 2]-\left(k_{\mathrm{Apcdiss}}+k_{\mathrm{Apci}}+k_{\mathrm{Cdc} 20 \mathrm{i}} \cdot[\mathrm{Cdk} 1: \mathrm{CycB}]\right.$ $+k_{\text {Emi2ass }} \cdot[$ Emi2 $\left.]\right) \cdot[$ Cdc20:APC $-\mathrm{P}]$

(7) $\frac{\mathrm{d} \mid \mathrm{Cdc} 20: \mathrm{APC}-\mathrm{P}: \text { Emi2 }]}{\mathrm{dt}}=k_{\text {Emizass }} \cdot[\mathrm{Emi} 2] \cdot[\mathrm{Cdc} 20: \mathrm{APC}-\mathrm{P}]-\left(k_{\text {Emi2diss }}+k_{\text {Emi2i }}\right.$ $\times[\mathrm{Cdk} 1: \mathrm{CycB}]) \cdot[\mathrm{Cdc} 20: \mathrm{APC}-\mathrm{P}: \mathrm{Emi} 2]$

$-\left(k_{\mathrm{Apci}}+k_{\mathrm{Cdc} 20 \mathrm{i}} \cdot[\mathrm{Cdk} 1: \mathrm{CycB}]\right) \cdot[\mathrm{Cdc} 20: \mathrm{APC}-\mathrm{P}: \mathrm{Emi} 2]$

(8) $[$ APC-P $]=[$ APC-P $]-[C d c 20: A P C-P]-[C d c 20: A P C-P: E m i 2]$

(9) $[$ Cdc20 $]=\left[\right.$ Cdc20 $\left.{ }_{T}^{\text {PP }}\right]-[$ Cdc20:APC-P $]-[C d c 20: A P C-P: E m i 2]$

(10) $[$ Emi2 $]=\left[\right.$ Emi2 $\left.{ }_{T}^{A}\right]-[$ Cdc20:APC-P:Emi2 $]$
Cdc20 phosphorylation but it is faster than the rate of $\mathrm{APC} / \mathrm{C}$ phosphorylation. As Emi2 gets inactivated, the concentration of Cdc20: APC-P complex starts to increase gradually which limits the rise in Cdk1:CycB activity. Once Cdc20:APC-P reaches a threshold the level of Cyclin B starts to decrease which further accelerates Cdc20:APC-P complex formation and Cyclin B degradation. Cdc20:APC-P gets inactivated by Emi2 once Cdk1:CycB activity drops to very low values. Furthermore, Cyclin B increases to a higher level in the presence of Emi2 since the rate of Cyclin B degradation is decreased by Emi2. The higher level of Cdk1: $\mathrm{CycB}$ in turn results in higher level of Cdc20:APC-P complex during the oscillations. The model simulation is consistent with the experimental observation reported for Cyclin B. Comparison of simulations on Fig. 2A and $B$ reveals that the inactivation of Cdc20:APC-P is much slower and incomplete without Emi2. Thus we conclude that $\mathrm{Emi} 2$ contributes to the dynamics of Cyclin B oscillations by effectively accelerating Cdc20: APC-P inactivation.

\subsection{Sensitivity to Emi2 levels}

Next we address the question of how sensitive are the amplitude and the period of Cyclin B oscillation to Emi2 levels? To illustrate this sensitivity, one parameter bifurcation diagrams is the obvious choice. A one-parameter bifurcation diagram illustrates the time-invariant solutions of a dynamical system as a function of an arbitrary chosen kinetic parameter [26]. A time-invariant solution could be a stable or unstable steady state or a limit cycle oscillation. A limit cycle oscillation 
A
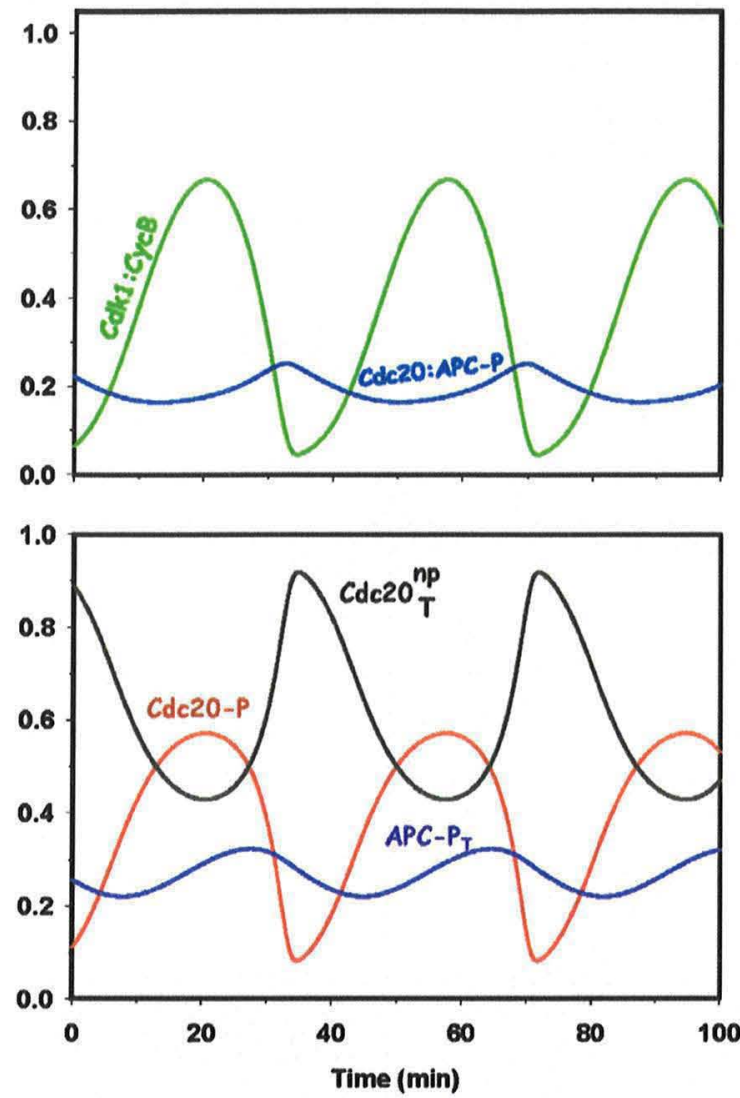

B
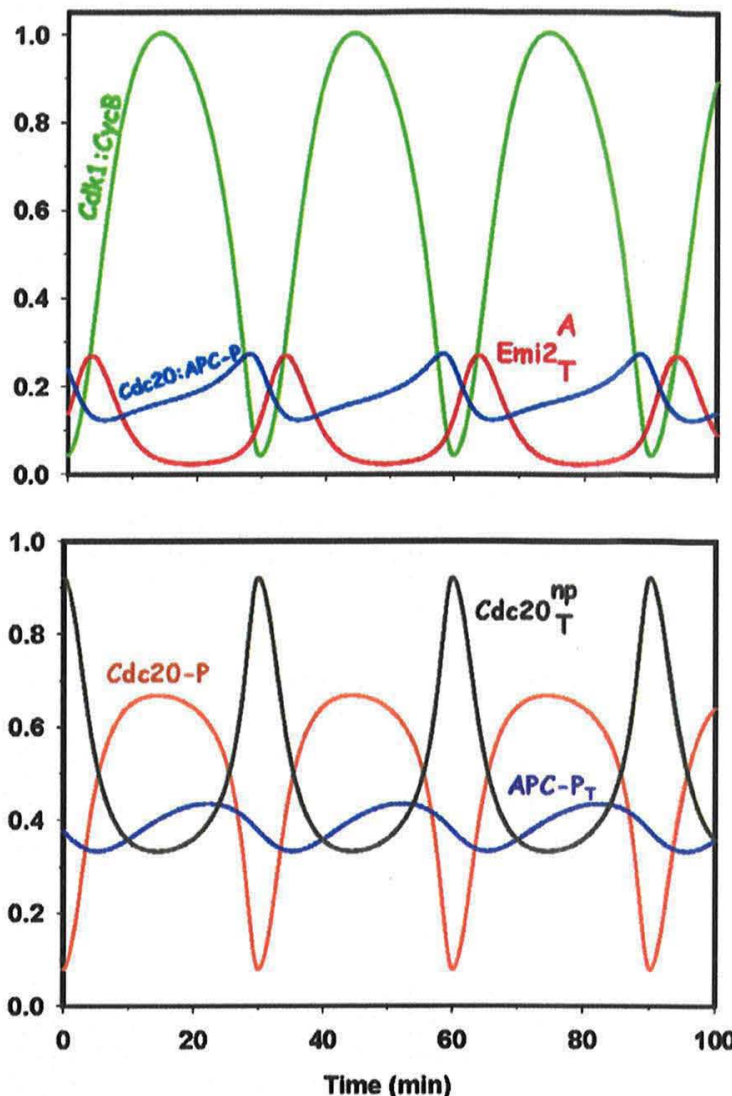

Fig. 2. Temporal simulation of network to demonstrate the effect of Emi2. (A) Limit cycle oscillations obtained using Ciliberto's model without the regulation of inhibitory Cdk1 phosphorylation. (B) Integration of Emi2 regulation into Ciliberto's model alters the characteristic of limit cycle oscillations. Abbreviations: Cdk1:CycB $=$ Cdk1 complex with CycB; Cdc20:APC-P = Cdc20 complex with APC-P; Cdc20-P = phosphorylated Cdc20; Cdc20 ${ }_{T}^{m p}=$ sum of Cdc20 unphosphorylated forms; APC-PT = sum of APC/C phosphorylated forms; $E m i 2 A=$ sum of Emi2 unphosphorylated (active) forms.

always emerges around unstable steady states (focus points) and can be characterized by the minima and maxima of the oscillation. It can emerge and disappear with a parameter change at so called bifurcation points. Since total level of Emi2 is constant during early embryonic cel cycles we choose it as a bifurcation parameter. We characterize the effect of Emi2 level on the oscillations by plotting the steady state Cyclin $\mathrm{B}$ levels and the minima and maxima of Cyclin B oscillations (Fig. 3A).

In the absence of Emi2 $\left(\mathrm{Emi}_{\mathrm{T}}=0\right)$ the network is identical to Ciliberto's model that gives rise to oscillations as indicated by the dots above and below the unstable steady state. The period of oscillation is plotted on Fig. 3B. As Emi2 level is increased the amplitude of oscillation increases in parallel with decrease in period since Emi2 accelerates Cdc20:APC-P inactivation. Once Emi2 level exceeds one unit, the period starts to extend slightly while amplitude increases further. The period extension is caused by Emi2 inhibition of Cdc20: APC-P activation. At Emi2 level around two, the amplitude drops abruptly and the oscillation disappears by a sub-critical Hopf bifurcation.

Table 2

List of kinetic parameters.

\begin{tabular}{|c|c|c|c|}
\hline \multicolumn{4}{|c|}{ Rate constant $\left(\mathrm{min}^{-1}\right)$} \\
\hline$k_{\text {cycs }}=0.4$ & $k_{\text {cycd }}=0.08$ & $k_{\text {cycd }} \cdot=2$ & $k_{\text {Apca }}=0.04$ \\
\hline$k_{\text {Apci }}=0.04$ & $k_{\mathrm{Cdc20a}}=5$ & $k_{\mathrm{CdC2Oi}}=10$ & $k_{\text {Apcass }}=100$ \\
\hline$k_{\text {Apcdiss }}=10$ & $k_{\mathrm{Emi2a}}=0.5$ & $k_{\mathrm{Emi2i}}=0.4$ & $k_{\text {Emizass }}=200$ \\
\hline$k_{\text {Emi2dis }}=20$ & $k_{\mathrm{Pp} 2 \text { a }}=0.05$ & $k_{\mathrm{Pp} 2 \mathrm{ai}}=0.001$ & $k_{\text {Pp2ai }} \cdot=3$ \\
\hline \multicolumn{4}{|c|}{ Other constants (dimensionless) } \\
\hline $\begin{array}{l}\mathrm{APC}_{\mathrm{T}}=1 \\
J_{\mathrm{Cyc}}=0.01\end{array}$ & $\mathrm{Cdc} 20_{\mathrm{T}}=1$ & $\mathrm{PP} \mathrm{A}_{\mathbf{T}}=1$ & $\operatorname{Emi} 2_{\mathrm{T}}=1$ \\
\hline
\end{tabular}

At the bifurcation point the unstable steady state gets replaced by a unique stable steady state and the period curve is also terminated at values observed in the absence of Emi2. At Emi2 levels larger than the bifurcation point, the limit cycle oscillation disappears, since Cdc20: APC-P activation is blocked by Emi2. We conclude that the model predicts a robust increase of Cyclin B amplitude with increasing Emi2 levels and arrest of embryos in high Cyclin B state with overexpression of Emi2. In contrast, the period of Cyclin B oscillation shows a minimum as a function of Emi2 levels but it is always smaller than the period of oscillation in the absence of Emi2.

The model presented here provides a potential explanation for the recently observed effect of Emi2 on the amplitude and the period of early embryonic cell cycle in Xenopus. The observed increase in amplitude and decrease in period of Cyclin B oscillation caused by Emi2 seem to be counter-intuitive at first sight: if the oscillation is faster we would expect smaller amplitude. Emi2 increases the amplitude of Cyclin B oscillation by inhibiting Cdc20:APC-P complexes which allows Cyclin $B$ to rise to a higher level during the oscillation. The period of oscillation decreases as a consequence of speeding up Cdc20:APC-P inactivation. This acceleration of Cdc20:APC-P inactivation is cancelled out at higher level of Emi2 by the inhibition of Cdc20: APC-P activation. These results suggest that altering Emi2 levels can fine tune the characteristic of early embryonic cell cycle by interfering with the intrinsic time delay in $\mathrm{APC} / \mathrm{C}$ activation and inactivation.

We conclude that the solution of the paradoxical experimental observations depends more on how Emi2 is regulated than the mechanism that generates oscillations in the model. The feed-forward regulation of Emi2 by $\mathrm{Cdk} 1: \mathrm{CycB}$ is one of the mechanisms that is 

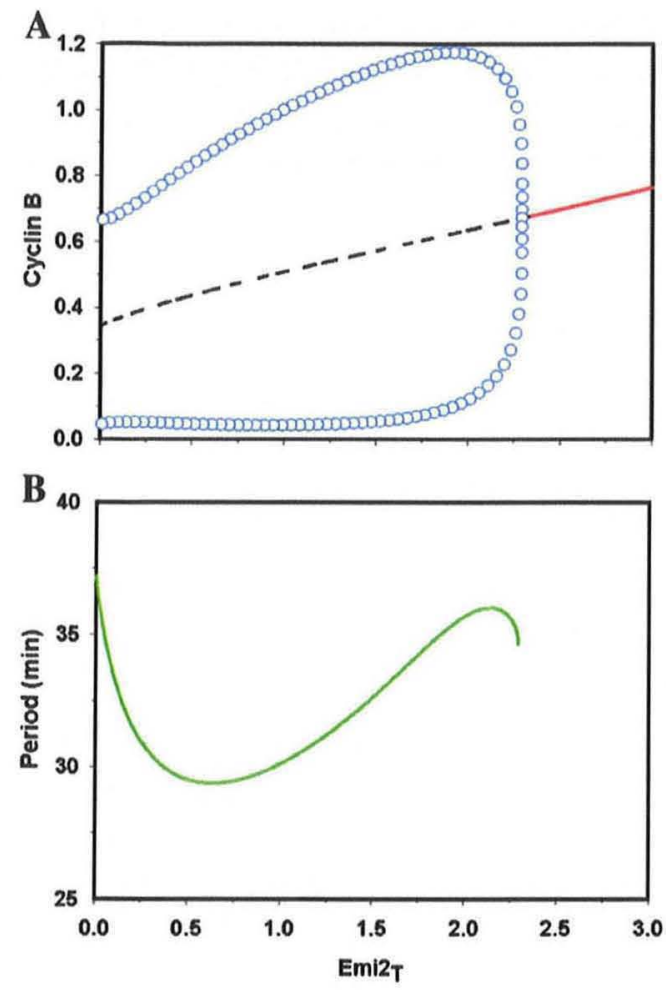

Fig. 3. Sensitivity of Cyclin B oscillations to Emi2 levels. (A) Bifurcation analysis of the model using $\mathrm{Emi} \mathrm{T}_{\mathrm{T}}$ as control parameter demonstrates a sub-critical Hopf bifurcation. The amplitude of oscillation (blue circles) increases with Emi2 levels until the bifurcation point where the unstable steady state surrounded by a limit cycle oscillation is replaced by a stable steady state (red solid line). (B) The period of oscillation vary depending upon the Emi2 levels.

sufficient to account for the experimental observations, because it provides the required non-linearity for $\mathrm{Cdk} 1 \mathrm{CycB}$ dependent regulation of Emi2.

\section{Numerical methods}

\subsection{Simulations and bifurcation analysis}

The network in Fig. 1 is translated into set of non-linear ordinary differential equations (ODEs) which describe the rate of change of concentration with time (Table 1). The equations are solved numerically with XPPAUT (available from G. Bard Ermentrout, University of Pittsburgh, PA, USA; http://www.math.pitt.edu/ bard/xpp/xpponw95. $\mathrm{html}$ ) to generate the temporal profile and bifurcation diagram.

Cdk1 activity depends on Cyclin B synthesis and degradation. The degradation of Cyclin B is catalysed by Cdc20:APC-P, a complex involving phosphorylated APC/C (APC-P) and dephosphorylated Cdc20 (Cdc20). Cyclin B degradation is described by Michaelis-Menten kinetics, while all other reactions are approximated by law of mass action. APC/C and Cdc20 are phosphorylated by $\mathrm{Cdk} 1: \mathrm{CycB}$ and dephosphorylated by constant phosphatase activities. APC/C inhibitor Emi2 is also phosphorylated by Cdk1:CycB and dephosphorylated by a Cdk1:CycB regulated phosphatase PP2A. We assume that the active form of Emi2 (dephosphorylated, Emi2 ${ }^{A}$ ) binds only to Cdc20:APC-P and form an inactive complex (Cdc20:APC-P:Emi2). We also take into consideration that the complexes (Cdc20:APC-P and Cdc20:APC-P:Emi2) dissociate by any of these scenarios: inhibitory phosphorylation of $\mathrm{Cdc} 20$, inhibitory phosphorylation of Emi2 and APC/C dephosphorylation. The model parameter values were obtained after iteratively testing different possible parameter sets based on Ciliberto et al. [12] to capture the oscillatory characteristic (amplitude and period) of early embryonic cell cycle in the presence and absence of Emi2. The counter-intuitive experimental observations allow us to restrict the parameter space to values given in Table 2 .

\section{Acknowledgements}

T.U.M is supported by the Collaborative Research Center 969 of the German Research Foundation (DFG). The work in B.N.'s group is supported by European Community's Seventh Framework Programme MitoSys (241548).

\section{References}

[1] T. Gotoh, LM. Villa, D.G. Capelluto, C.V. Finkielstein, Regulatory pathways coordinating cell cycle progression in early Xenopus development, Results and Problems in Cell Differentiation 53 (2011) 171-199.

[2] M.S. Murakami, G.F. Vande Woude, Analysis of the early embryonic cell cycles of Xenopus; regulation of cell cycle length by Xe-wee1 and Mos, Development 125 (1998) 237-248.

[3] R.S. Hartley, R.E. Rempel, J.L. Maller, In vivo regulation of the early embryonic cell cycle in Xenopus, Developmental Biology 173 (1996) 408-419.

[4] J.E. Ferrell Jr., M. Wu, J.C. Gerhart, G.S. Martin, Cell cycle tyrosine phosphorylation of p34cdc2 and a microtubule-associated protein kinase homolog in Xenopus oocytes and eggs, Molecular and Cellular Biology 11 (1991) 1965-1971.

[5] S.H. Kim, C. Li, J.L. Maller, A maternal form of the phosphatase Cdc25A regulates early embryonic cell cycles in Xenopus laevis, Developmental Biology 212 (1999) 381-391.

[6] J.A. Pesin, T.L. Orr-Weaver, Regulation of APC/C activators in mitosis and meiosis, Annual Review of Cell and Developmental Biology 24 (2008) 475-499.

[7] J.M. Peters, The anaphase promoting complex/cyclosome: a machine designed to destroy, Nature Reviews. Molecular Cell Biology 7 (2006) 644-656.

[8] T. Lorca, A. Castro, A.M. Martinez, S. Vigneron, N. Morin, S. Sigrist, C. Lehner, M. Doree, J.C. Labbe, Fizzy is required for activation of the APC/cyclosome in Xenopus egg extracts, The EMBO Journal 17 (1998) 3565-3575.

[9] Y. Zhou, Y.P. Ching, R.W. Ng, D.Y. Jin, The APC regulator CDH1 is essential for the progression of embryonic cell cycles in Xenopus, Biochemical and Biophysical Research Communications 294 (2002) 120-126.

[10] H. Labit, K. Fujimitsu, N.S. Bayin, T. Takaki, J. Gannon, H. Yamano, Dephosphorylation of Cdc20 is required for its C-box-dependent activation of the APC/C, The EMBO Journal 31 (2012) 3351-3362.

[11] V. D'Angiolella, C. Mari, D. Nocera, L. Rametti, D. Grieco, The spindle checkpoint requires cyclin-dependent kinase activity, Genes \& Development 17 (2003) 2520-2525.

[12] A. Ciliberto, A. Lukacs, A. Toth, J.J. Tyson, B. Novak, Rewiring the exit from mitosis, Cell Cycle 4 (2005) 1107-1112.

[13] K. Ohsumi, A. Koyanagi, T.M. Yamamoto, T. Gotoh, T. Kishimoto, Emi1-mediated $\mathrm{M}$-phase arrest in Xenopus eggs is distinct from cytostatic factor arrest, Proceedings of the National Academy of Sciences of the United States of America 101 (2004) $12531-12536$

[14] J. Minshull, H. Sun, N.K. Tonks, A.W. Murray, A MAP kinase-dependent spindle assembly checkpoint in Xenopus egg extracts, Cell 79 (1994) 475-486.

[15] A. Schmidt, P.I. Duncan, N.R. Rauh, G. Sauer, A.M. Fry, E.A. Nigg, T.U. Mayer, Xenopus polo-like kinase Plx1 regulates XErp1, a novel inhibitor of APC/C activity, Genes \& Development 19 (2005) 502-513.

[16] J.J. Tung, D.V. Hansen, K.H. Ban, A.V. Loktev, M.K. Summers, J.R. Adler III, P.K. Jackson, $A$ role for the anaphase-promoting complex inhibitor Emi2/XErp1, a homolog of early mitotic inhibitor 1 , in cytostatic factor arrest of Xenopus eggs, Proceedings of the National Academy of Sciences of the United States of America 102 (2005) 4318-4323.

[17] N.R. Rauh, A. Schmidt, J. Bormann, E.A. Nigg, T.U. Mayer, Calcium triggers exit from meiosis II by targeting the APC/C inhibitor XErp1 for degradation, Nature 437 (2005) $1048-1052$

[18] D.V. Hansen, J.J. Tung, P.K. Jackson, CaMKII and polo-like kinase 1 sequentially phosphorylate the cytostatic factor Emi2/XErp1 to trigger its destruction and meiotic exit, Proceedings of the National Academy of Sciences of the United States of America 103 (2006) 608-613.

[19] J. Liu, B. Grimison, A.L. Lewellyn, J.L. Maller, The anaphase-promoting complex/cyclosome inhibitor Emi2 is essential for meiotic but not mitotic cell cycles, The Journal of Biological Chemistry 281 (2006) 34736-34741.

[20] T. Tischer, E. Hormanseder, T.U. Mayer, The APC/C inhibitor XErp1/Emi2 is essential for Xenopus early embryonic divisions, Science 338 (2012) 520-524.

[21] B. Novak, J.J. Tyson, Design principles of biochemical oscillators, Nature Reviews. Molecular Cell Biology 9 (2008) 981-991.

[22] M.H. Schmitz, M. Held, V. Janssens, J.R. Hutchins, O. Hudecz, E. Ivanova, J. Goris, L. Trinkle-Mulcahy, A.I. Lamond, I. Poser, A.A. Hyman, K. Mechtler, J.M. Peters, D.W. Gerlich, Live-cell imaging RNAi screen identifies PP2A-B55alpha and importin-beta 1 as key mitotic exit regulators in human cells, Nature Cell Biology 12 (2010) 886-893. 
[23] J.Q.Wu, J.Y. Guo, W. Tang, C.S. Yang, C.D. Freel, C. Chen, A.C. Nairn, S. Kornbluth PPl-mediated dephosphorylation of phosphoproteins at mitotic exit is controlled by inhibitor-1 and PP1 phosphorylation, Nature Cell Biology 11 (2009) 644-651

[24] S. Mochida, S.L. Maslen, M. Skehel, T. Hunt, Greatwall phosphorylates an inhibito of protein phosphatase 2A that is essential for mitosis, Science 330 (2010) $1670-1673$.
[25] A. Gharbi-Ayachi, J.C. Labbe, A. Burgess, S. Vigneron, J.M. Strub, E. Brioudes, A. Van-Dorsselaer, A. Castro, T. Lorca, The substrate of Greatwall kinase, Arpp19, controls mitosis by inhibiting protein phosphatase $2 \mathrm{~A}$, Science 330 (2010) 1673-1677.

[26] A. Csikasz-Nagy, D. Battogtokh, K.C. Chen, B. Novak, J.J. Tyson, Analysis of a generic model of eukaryotic cell-cycle regulation, Biophysical Journal 90 (2006) 4361-4379. 